\title{
ISLA - ERFOLG AUF ALLEN KONTINENTEN
}

\section{Liebe Kolleginnen und Kollegen,}

wieder einmal neigt sich ein Jahr zu Ende und gerade in den letzten Monaten sind sehr viele neue und spannende Dinge innerhalb der ISLA-transkontinental passiert.

Es ist der ISLA gelungen, in Kooperation mit der Pharmakologie der Universität Marburg in Deutschland ein neues Indozyaningrünpräparat in Nanopartikeln zu entwickeln, das nach den ersten Behandlungen möglicherweise einen Durchbruch in der photodynamischen Tumortherapie bringen wird. Erste Behandlungen sind sehr vielversprechend. Diese ersten Resultate wurden auf der 4. ISLA-Asienkonferenz in Bangkok vom 29.11.-1.12.2018 vorgestellt. Die Konferenz brachte 130 TeilnehmerInnen aus fast allen asiatischen Ländern zusammen, und zusätzlich kam auch eine Reihe KollegenInnen aus Europa, den USA, Südamerika, Südafrika und Australien hinzu. Es waren 25 Redner aus allen Erdteilen mit exzellenten neuen Behandlungsstrategien und wissenschaftlichen Grundlagenforschungen vertreten. So wird deutlich, wie sehr sich die ISLA und ihre Themen immer mehr weltweit verbreiten.

Während der Konferenz wurde ein Vertrag über die Gründung der ISLA Japan von Prof. Litscher und Dr. Weber unterzeichnet und ein erster ISLA-Kongress in Tokio für den 17. März 2019 geplant.

So wird es im Jahr 2019 vier internationale ISLAKonferenzen geben: Ende Januar die 2. USA-Konferenz

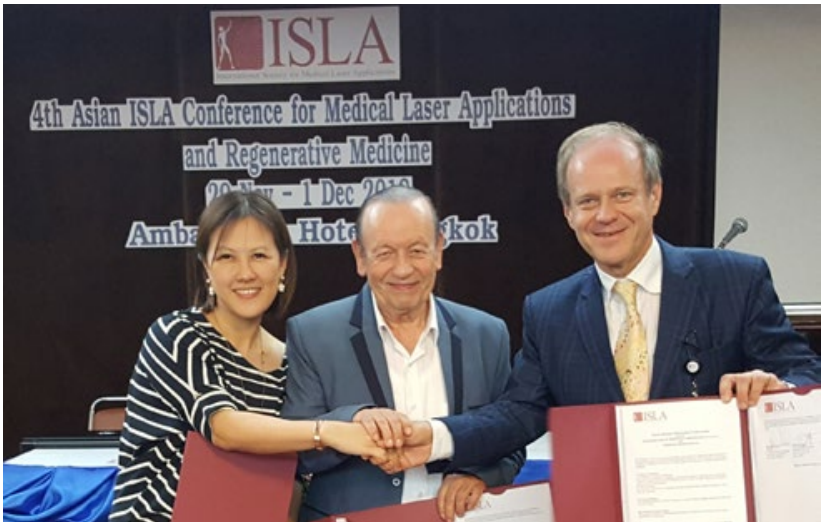

Abb. 1: Lin Cheng Wen, Vice-President CBP Tokyo, Japan, Dr. Michael Weber, Prof. DDr. Gerhard Litscher (v.l.n.r.), Bangkok, 30. November 2018

in San Diego, am 17. März die 1. Japan-Konferenz in Tokio, Ende Juni der 15. ISLA-Deutschland-Kongress in Beverungen und Ende November der bereits 5. in Bangkok stattfindende Asienkongress.

Wir hoffen sehr, dass Sie alle eine dieser Konferenzen besuchen werden. Hauptthemen werden wieder Schmerztherapie, Akupunktur, regenerative Medizin, laserstimulierte Stammzelltherapie und photodynamische Tumortherapie sein.

Wir wünschen allen ein schönes Weihnachtsfest und ein glückliches und erfolgreiches Jahr 2019.

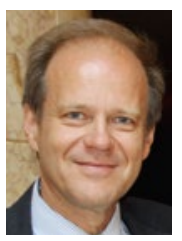

Univ.-Prof. Prof. h.c. DI Dr. techn.

Dr. scient. med.Gerhard Litscher

President for Science and Research der International Society for Medical Laser Applications (ISLAtranscontinental)

Medizinische Universität Graz, Auenbruggerplatz 39, EG19, A-8036 Graz, Tel: +43 316/385-83907

E-Mail:gerhard.litscher@medunigraz.at,Internet:http://litscher.info 